\title{
Manejo da água na irrigação da alfafa num Latossolo Vermelho-Amarelo(1)
}

\author{
Joaquim Bartolomeu Rassini(2)
}

\begin{abstract}
Resumo - O objetivo deste trabalho foi avaliar um método prático para manejar a água de irrigação na cultura da alfafa (Medicago sativa). O estudo foi desenvolvido em um Latossolo Vermelho-Amarelo, e as variáveis estudadas foram: a evaporação do tanque classe A (ECA), a precipitação pluvial (PRP) e a capacidade do solo de armazenar água (CAD). Os tratamentos constituíram-se de três regimes hídricos aplicados: $\mathrm{H}_{1}$ : testemunha, sem irrigação; $\mathrm{H}_{2}$ : irrigação complementar em um determinado período da cultura, quando ECA - PRP $\geq 30 \mathrm{~mm}$, a partir da emissão do primeiro afilho secundário; $\mathrm{H}_{3}$ : irrigação complementar em determinado período da cultura, quando ECA - PRP $\geq 20 \mathrm{~mm}$, durante todo o ciclo da planta. Verificou-se que o método ECA - PRP $=20$ a $30 \mathrm{~mm}$ é compatível com a CAD desse solo, e que também pode-se aumentar a eficiência da água aplicada na alfafa sem provocar queda no rendimento de forragem
\end{abstract}

Termos para indexação: Medicago sativa, rendimento, balanço hídrico, precipitação, retenção de água pelo solo.

\section{Water management of irrigation for alfalfa in a Red-Yellow Latosol (Hapludox)}

\begin{abstract}
This work was carried out to evaluate a practical method for the irrigation water management for alfalfa (Medicago sativa) cultivation. The study was conducted in a Red-Yellow Latosol (Hapludox) soil, and the variables studied were: "A" tank evaporation (CAE), rainfall $(\mathrm{R})$ and water storage capacity of the soil (WSC). Treatments consisted of three hydric conditions: $\mathrm{H}_{1}$ : no irrigation; $\mathrm{H}_{2}$ : supplement irrigation when CAE - $\mathrm{PRP} \geq 30 \mathrm{~mm}$ from full vegetative stage on plant; $\mathrm{H}_{3}$ : supplement irrigation when $\mathrm{CAE}-\mathrm{PRP} \geq 20 \mathrm{~mm}$ during entire plant cycle. It was found that the method for the Red-Yellow Latosol using CAE - R $=20$ to $30 \mathrm{~mm}$ is consistent with the WSC, and that efficiency of utilization usage of water applied can be increased, without decreasing the forage yield.
\end{abstract}

Index terms: Medicago sativa, yields, water balance, precipitation, soil water retention

\section{Introdução}

Na agricultura, a irrigação é fundamental para eliminar o risco de deficiência hídrica nos cultivos, aumentar a produtividade, garantir a produção de alimentos e intensificar o uso da terra. Por outro lado, a elevada demanda de água em projetos de irrigação no Brasil exige racionalização no seu uso por meio de alternativas que proporcionem maior eficiência no uso da água.

O clima é o fator abiótico que mais afeta a perda de água pela planta. Outros fatores, como o solo, as pragas, as doenças e a própria cultura, além das práticas agrícolas, também influenciam a transferência

(1) Aceito para publicação em 3 de julho de 2001

(2) Embrapa-Centro de Pesquisa de Pecuária do Sudeste, Caixa Postal 339, CEP 13560-970 São Carlos, SP. E-mail rassini@cppse.embrapa.br da água para a atmosfera. No entanto, sua medição em práticas de manejo de irrigação, por evapotranspiração, se baseia em inúmeras fórmulas que envolvem dois ou mais parâmetros climáticos e índices ligados às espécies vegetais. Klar (1991) descreve esses métodos de perda de água das culturas, como os hidrológicos ou de balanço de água, os que envolvem correções turbulentas, os de balanço de energia, os combinados que conjugam partes do balanço de energia e de transporte de massa, os empíricos de Thorntwaite e Blaney - Criddle, e a utilização de evaporímetros, como o tanque classe A (ECA), o qual envolve um complexo de fatores ligados à posição do tanque $(\mathrm{Kp})$ e a própria planta $(\mathrm{Kc})$. Segundo esse mesmo autor, o tensiômetro, que mede diretamente o potencial matricial da água do solo, é confiável até valores em torno de $-0,75$ bar, pois trabalha sob vácuo, e devido às imperfeições mecânicas seu uso não permite que a máxima sucção teórica 
(valor negativo da pressão atmosférica local) seja atingida. Além disso, na prática, em razão de diferenças até pontuais nas propriedades físicas dos solos, há influência na decisão de se irrigar, ou seja, é comum a ocorrência de potenciais de água no solo de dois tensiômetros em uma área e na mesma profundidade sugerir ao mesmo tempo a aplicação e não-aplicação da água.

Todas essas dificuldades têm levado os irrigantes a adotar o manejo de maior erro do ponto de vista técnico, econômico e ecológico, que é a aplicação da água com base em freqüências e lâminas de irrigação predeterminadas, ou seja, para cada $5,6,7,8$ ou 10 dias sem chuvas (freqüências), aplicam-se, respectivamente, 5, 10, 15, 20 ou $25 \mathrm{~mm}$ de água (lâminas). Essa prática não ocorre apenas em nossas condições, pois na Índia, onde a irrigação é muito utilizada, diversos trabalhos foram realizados visando aumentar a eficiência do uso da água, por meio de métodos de fácil aplicação (Hukkeri et al., 1977; Gill \& Malik, 1983; Lal, 1987). Com relação ao aumento da eficiência no uso da água de irrigação, Gilley et al. (1981), nos EUA, verificaram que, diminuindo ou mesmo eliminando a irrigação em alguns estádios das culturas, não havia queda substancial da produção. No Brasil, resultados semelhantes foram obtidos por Rassini \& Leme (2001), os quais constataram que a água aplicada no início do estádio vegetativo da alfafa não aumentou o rendimento de forragem, determinando menor desenvolvimento radicular.

O objetivo deste trabalho foi avaliar um método prático de manejar a água de irrigação na cultura da alfafa.

\section{Material e Métodos}

O trabalho foi realizado no período de junho de 1999 a junho de 2000, num Latossolo Vermelho-Amarelo de textura média e relevo plano, na Embrapa-Centro de Pesquisa de Pecuária do Sudeste, São Carlos, SP, com as seguintes características físico-químicas (0 a $20 \mathrm{~cm}$ ): argila, $279 \mathrm{~g} \mathrm{~kg}^{-1}$, macroporos, $15 \%$; condutividade hidráulica, $3,8 \mathrm{~m} \mathrm{dia}^{-1}$, taxa de infiltração, $273 \mathrm{~mm} \mathrm{hora}^{-1}$; $\mathrm{pH}$ em $\mathrm{H}_{2} \mathrm{O}, 6,6 ; \mathrm{pH}$ em $\mathrm{CaCl}_{2}, 6,2 ; \mathrm{MO}, 17 \mathrm{~g} \mathrm{dm}^{-3} ; \mathrm{P}, 13 \mathrm{mg} \mathrm{dm}^{-3}$. $\mathrm{K}, \quad 3,4 \mathrm{mmol}_{\mathrm{c}} / \mathrm{dm}^{-3} ; \quad \mathrm{Ca}, 40 \mathrm{mmol}_{\mathrm{c}} / \mathrm{dm}^{-3} ; \mathrm{Mg}$, $18 \mathrm{mmol}_{\mathrm{c}} / \mathrm{dm}^{-3}$; $\mathrm{Al}, 1 \mathrm{mmol}_{\mathrm{c}} / \mathrm{dm}^{-3}$; CTC, $80 \%$ e V, $77 \%$ (Calderano Filho et al., 1998)

Utilizou-se a alfafa cultivar Crioula e a semeadura foi realizada em 7/6/1999, conforme Rassini (1998)
Os tratamentos constituíram-se de três regimes hídricos: $\mathrm{H}_{1}$ : testemunha sem irrigação complementar; $\mathrm{H}_{2}$ : irrigação complementar quando a diferença entre os valores acumulados do tanque classe A (ECA) e da precipitação pluvial (PRP), a partir da emissão do primeiro afilho secundário da alfafa, em um determinado período, fosse igual ou maior que $30 \mathrm{~mm}$ (ECA - $\mathrm{PRP} \geq 30 \mathrm{~mm}) ; \mathrm{H}_{3}$ : irrigação complementar durante todo o ciclo da cultura, quando em determinado período, ECA - PRP $\geq 20 \mathrm{~mm}$. As avaliações de rendimento de forragem foram obtidas em unidades experimentais de $6 \mathrm{~m}^{2}$ de área útil. Do material verde obtido nessa avaliação, retirou-se uma amostra que foi pesada e secada a $70^{\circ} \mathrm{C}$, por 72 horas, para determinação da matéria seca. Foi determinado o peso seco de raízes de cinco plantas por parcela no primeiro e no último corte, utilizandose o mesmo método

A irrigação foi realizada por meio de um aspersor de giro completo, de bocal duplo Modelo ZED 30 da ASBRASIL, dotado de um tubo de subida de $0,5 \mathrm{~m}$, com espaçamento de 7,0 m entre os aspersores instalados numa linha longitudinal que passava pelo eixo central da área experimental. Segundo Primavesi et al. (1999), a capacidade de armazenamento de água (CAD) do solo na camada de 0 a $1,0 \mathrm{~m}$ é de $49 \mathrm{~mm}$ e na camada de 0 a $0,2 \mathrm{~m}$ varia de 15,0 a $24,5 \mathrm{~mm}$, sendo que o ponto de murcha permanente (PMP) correspondente a uma tensão de 1,5 $\mathrm{MPa}$ é de 6,6 a $11,7 \%$ em água. Antes da instalação dos tratamentos, o equipamento de irrigação foi testado por meio de quatro pluviômetros em cada parcela, verificando-se que um tempo de 150 minutos era coerente com as condições físicohídricas do solo. Durante as irrigações, determinou-se o conteúdo de água de três amostras de solo $(0$ a $0,1 \mathrm{~m})$ por gravimetria, coletadas antes e logo após a aplicação da água.

O balanço hídrico simplificado baseou-se em valores coletados diariamente da evaporação do tanque classe $\mathrm{A}$, e de seis pluviômetros instalados na área experimental. Quando os dados atingiam as condições previamente estabelecidas nos tratamentos $\mathrm{H}_{2} \mathrm{e} \mathrm{H}_{3}$, realizava-se a irrigação complementar

O delineamento experimental usado foi o de blocos ao acaso, com três repetições. A análise de variância do rendimento de forragem e do desenvolvimento radicular foi realizada conforme Pimentel-Gomes (1978) e as médias comparadas pelo teste de Tukey a $1 \%$ de probabilidade

\section{Resultados e Discussão}

A precipitação pluvial de $1.585,0 \mathrm{~mm}$, acumulada durante o período experimental, foi superior em 226,6 mm à evaporação normalmente registrada nes- 
se período. Porém, sua distribuição foi muito desuniforme, concentrando-se nos meses de dezembro de 1999, janeiro, fevereiro e março de 2000. Nos meses de julho, agosto, setembro, outubro, novembro de 1999, abril, maio e junho de 2000 foi inferior, com um déficit de 628,6 mm em relação à evaporação. Esses resultados estão de acordo com Primavesi et al. (1999).
Não houve grandes diferenças entre as lâminas de irrigação (LAI), sendo em média de 19,0 mm no tratamento $\mathrm{H}_{3}$ e de $17,7 \mathrm{~mm}$ no $\mathrm{H}_{2}$ (Tabela 1). O conteúdo de água do solo, antes e após as irrigações, apresentou valores bem próximos aos de Primavesi et al. (1999), para o mesmo solo: 7,1\% antes da irrigação (Ai) e 16,9\% depois da irrigação (Di). Frizzone et al. (1995), no Brasil, manejando água de

Tabela 1. Manejo de irrigação em alfafa, por meio da evaporação do tanque classe A (ECA) e da precipitação pluvial (PRP), em diferentes condições de umidade do solo, em 1999.

\begin{tabular}{|c|c|c|c|c|c|c|c|c|c|}
\hline \multirow{2}{*}{$\begin{array}{l}\text { Dias após } \\
\text { tratamento } \\
\text { (dias) }\end{array}$} & \multicolumn{3}{|c|}{$\mathrm{H}_{3}(\mathrm{ECA}-\mathrm{PRP} \geq 20 \mathrm{~mm})^{(1)}$} & \multicolumn{3}{|c|}{$\mathrm{H}_{2}(\text { ECA-PRP } \geq 30 \mathrm{~mm})^{(2)}$} & \multicolumn{3}{|c|}{ Umidade do solo (\%) } \\
\hline & $\begin{array}{c}\text { ECA-PRP } \\
(\mathrm{mm})\end{array}$ & $\begin{array}{c}\mathrm{FRQ}^{(3)} \\
\text { (dias) }\end{array}$ & $\begin{array}{l}\mathrm{LAI}^{(4)} \\
(\mathrm{mm})\end{array}$ & $\begin{array}{c}\text { ECA-PRP } \\
(\mathrm{mm})\end{array}$ & $\begin{array}{c}\mathrm{FRQ}^{(3)} \\
\text { (dias) }\end{array}$ & $\begin{array}{l}\mathrm{LAI}^{(4)} \\
(\mathrm{mm})\end{array}$ & $\mathrm{H}_{1}^{(5)}$ & $\mathrm{Ai}^{(6)}$ & $\mathrm{Di}^{(7)}$ \\
\hline 0 & - & - & 14,0 & - & - & - & 6,0 & 7,8 & 19,6 \\
\hline 12 & 24,6 & 12 & 23,8 & - & - & - & 6,2 & 8,3 & 20,5 \\
\hline 20 & 30,0 & 8 & 20,8 & 30,0 & 20 & 19,6 & 6,3 & 6,2 & $19,1^{(8)}$ \\
\hline 28 & 29,3 & 8 & 20,4 & - & - & - & 5,1 & 8,2 & 20,7 \\
\hline 30 & - & - & - & 35,8 & 10 & 20,0 & 5,1 & 7,8 & 17,4 \\
\hline 36 & 26,6 & 8 & 19,7 & - & - & - & 5,2 & 7,4 & 17,9 \\
\hline 38 & - & - & - & 35,6 & 10 & 18,8 & 5,1 & 5,9 & 18,2 \\
\hline 42 & 29,9 & 6 & 18,9 & - & - & - & 5,8 & 6,8 & 17,2 \\
\hline 46 & - & - & - & 36,3 & 8 & 22,9 & 3,6 & 5,6 & 20,2 \\
\hline 48 & 26,5 & 6 & 23,8 & - & - & - & 4,0 & 8,5 & 18,7 \\
\hline 54 & 26,8 & 6 & 16,8 & 35,8 & 8 & 16,3 & 3,5 & 7,0 & $15,2^{(8)}$ \\
\hline 59 & 28,8 & 5 & 19,6 & - & - & - & 4,7 & 6,6 & 16,6 \\
\hline 61 & - & - & - & 39,1 & 7 & 18,2 & 4,0 & 6,0 & 15,3 \\
\hline 64 & 32,1 & 5 & 15,6 & - & - & - & 4,4 & 6,9 & 16,0 \\
\hline 67 & - & - & - & 41,0 & 6 & 13,6 & 3,4 & 4,4 & 15,8 \\
\hline 68 & 26,0 & 4 & 15,4 & - & - & - & 4,6 & 7,0 & 17,4 \\
\hline 97 & 20,0 & 28 & 26,0 & - & - & - & 5,1 & 6,6 & 17,3 \\
\hline 98 & - & - & - & 30,0 & 30 & 25,0 & 5,5 & 5,8 & 17,2 \\
\hline 103 & 20,5 & 5 & 20,1 & - & - & - & 6,2 & 9,5 & 17,0 \\
\hline 106 & - & - & - & 33,1 & 9 & 18,7 & 5,1 & 6,8 & 17,6 \\
\hline 107 & 24,8 & 4 & 21,9 & - & - & - & 5,2 & 9,0 & 19,2 \\
\hline 117 & 26,1 & 10 & 19,0 & 33,1 & 11 & 18,9 & 5,7 & 6,7 & $16,4^{(8)}$ \\
\hline 125 & 24,6 & 8 & 20,2 & - & - & - & 5,6 & 5,8 & 17,2 \\
\hline 127 & - & - & - & 37,2 & 10 & 15,0 & 5,1 & 6,2 & 15,3 \\
\hline 155 & 20,3 & 28 & 22,0 & - & - & - & 7,2 & 8,1 & 15,0 \\
\hline 253 & - & 98 & 11,1 & - & 126 & 11,0 & 5,7 & 6,5 & $15,5^{(8)}$ \\
\hline 258 & 28,9 & 5 & 17,3 & - & - & - & 5,8 & 7,4 & 16,0 \\
\hline 260 & - & - & - & 33,8 & 7 & 19,7 & 4,8 & 5,8 & 15,2 \\
\hline 263 & 20,2 & 5 & 19,7 & - & - & - & 6,1 & 7,8 & 16,8 \\
\hline 268 & - & - & - & 31,1 & 8 & 10,2 & 5,3 & 5,9 & 13,1 \\
\hline 269 & 26,0 & 6 & 12,6 & - & - & - & 4,8 & 6,2 & 14,4 \\
\hline 275 & 25,7 & 6 & 17,8 & 30,7 & 7 & 18,3 & 5,2 & 6,8 & $14,9^{(8)}$ \\
\hline 284 & 22,6 & 9 & 22,9 & - & - & - & 5,9 & 7,9 & 18,2 \\
\hline 288 & - & - & - & 37,0 & 13 & 19,0 & 5,6 & 6,0 & 14,5 \\
\hline 290 & 21,6 & 6 & 16,0 & - & - & - & 5,5 & 9,3 & 17,8 \\
\hline 298 & 22,9 & 8 & 17,5 & - & - & - & 6,0 & 9,8 & 16,8 \\
\hline 298 & - & - & - & 30,1 & 10 & 16,1 & 6,0 & 7,7 & 15,9 \\
\hline Média & 25,4 & 8 & 19,0 & 34,4 & 11 & 17,7 & 5,2 & 7,1 & 16,9 \\
\hline
\end{tabular}

${ }^{(1)} \mathrm{H}_{3}$ : irrigação complementar em determinado período da cultura, quando ECA - PRP $\geq 20 \mathrm{~mm}$; durante todo o ciclo da cultura, a lâmina total de irrigação foi de $472,9 \mathrm{~mm} .{ }^{(2)} \mathrm{H}_{2}$ : irrigação complementar aplicada após a emissão do lo afilho, quando ECA - PRP $\geq 30$ mm; a lâmina total de irrigação foi de 301,3 mm. ${ }^{(3)}$ Frequêencia de irrigação. ${ }^{(4)}$ Lâmina de irrigação. ${ }^{(5)}$ Sem irrigação. ${ }^{(6)}$ Umidade do solo antes da irrigação (\% em peso). ( ${ }^{(7)}$ Umidade do solo após a irrigação (\% em peso). ${ }^{(8)}$ Nos dias em que foram aplicadas irrigação nos dois tratamentos, os valores de Ai e Di se referem a média de $\mathrm{H}_{3}$ e $\mathrm{H}_{2}$. 
irrigação complementar em aveia forrageira (Avena sativa) num Latossolo Vermelho-Escuro, obtiveram resultados semelhantes usando o mesmo critério do tratamento $\mathrm{H}_{2}$, isto é, toda vez que a diferença entre a evaporação do tanque classe A (ECA) e as precipitações em determinado período da cultura atingia $30 \mathrm{~mm}$, era realizada a irrigação. Estudos realizados na Índia (Hukkeri et al., 1977; Lal, 1987) também corroboram os dados obtidos no presente trabalho, relacionando dados do tanque classe A e teor de água no solo, com produtividade de forragem de aveia.

$\mathrm{O}$ uso pleno da água em todo ciclo da alfafa $\left(\mathrm{H}_{3}\right)$ propiciou menor intervalo entre duas irrigações sucessivas, sendo em média de oito dias, com maior freqüência de irrigação (FRQ) (Tabela 1). Porém, com o uso mais eficiente da água de irrigação, a partir do estádio vegetativo pleno $\left(\mathrm{H}_{2}\right)$, o intervalo entre duas irrigações aumentou para 11 dias. Assim, com o manejo $\mathrm{H}_{3}$, aplicou-se uma lâmina total de $472,9 \mathrm{~mm}$ de água e com o manejo $\mathrm{H}_{2}$, foram aplicados $301,3 \mathrm{~mm}$ de água de irrigação complementar, com uma economia de $171,6 \mathrm{~mm}$ ou $1.716 .000 \mathrm{~L}$ de água por hectare.

Os valores médios das produtividades de matéria seca de forragem de alfafa em dez cortes e do total no ano e o peso do sistema radicular inicial $\left(\mathrm{C}_{1}\right)$ e final $\left(\mathrm{C}_{10}\right)$ foram afetados significativamente pelos tratamentos (Tabela 2).
Quanto à produção de forragem, os tratamentos $\mathrm{H}_{2}$ (17,5 t ha ${ }^{-1}$ ano $^{-1}$ de MS) e o $\mathrm{H}_{3}\left(15,1 \mathrm{t} \mathrm{ha}^{-1} \mathrm{ano}^{-1}\right.$ de MS) diferiram significativamente da testemunha $\mathrm{H}_{1}$ (11,4 t ha- ${ }^{-1}$ ano $^{-1}$ de MS), mas não entre si. Esses níveis de produção, bem como sua distribuição ao longo do ano, estão de acordo com os dados de Fontes et al. (1993), Santos (1993) e Rassini (1998). Em função da irrigação complementar, houve um incremento de $43 \%$ na produtividade de forragem de alfafa, ou seja, de 11,4 para 16,3 $\mathrm{t} \mathrm{ha}^{-1} \mathrm{ano}^{-1}$ de MS.

$\mathrm{O}$ desenvolvimento inicial e final do sistema radicular da alfafa foi bastante responsivo à água do solo, pois, quando não se aplicou irrigação complementar no início do desenvolvimento da planta, o peso de matéria seca das raízes foi de $2,89\left(\mathrm{H}_{1}\right)$ e $2,91 \mathrm{~g} \mathrm{planta}^{-1}\left(\mathrm{H}_{2}\right)$, sendo significativamente superiores ao do manejo $\mathrm{H}_{3}\left(2,16 \mathrm{~g} \mathrm{planta}^{-1}\right)$ no primeiro corte $\left(\mathrm{C}_{1}\right)$. Essa condição repetiu-se no último corte $\left(\mathrm{C}_{10}\right)$, quando o menor peso do sistema radicular foi registrado no tratamento $\mathrm{H}_{3}\left(30,53 \mathrm{~g} \mathrm{planta}^{-1}\right)$, que diferiu significativamente dos tratamentos $\mathrm{H}_{1}$ $\left(44,47 \mathrm{~g} \mathrm{planta}^{-1}\right)$ e $\mathrm{H}_{2}\left(41,56 \mathrm{~g} \mathrm{planta}^{-1}\right)$, os quais, por sua vez, não diferiram entre si. Rassini \& Leme (2001) também constataram que a água de irrigação aplicada no início do desenvolvimento da alfafa prejudicou o desenvolvimento radicular da planta e, conseqüentemente, reduziu o rendimento de forragem.

Tabela 2. Produtividade de forragem e desenvolvimento radicular da alfafa, em três regimes de irrigação: $\mathrm{H}_{1}$ : sem irrigação; $\mathrm{H}_{2}$ : irrigação complementar aplicada quando ECA - PRP $\geq 30 \mathrm{~mm} ; \mathrm{H}_{3}$ : irrigação complementar aplicada quando ECA - PRP $\geq 20 \mathrm{~mm}$.

\begin{tabular}{|c|c|c|c|c|c|c|c|c|c|c|c|c|}
\hline \multirow{3}{*}{$\begin{array}{c}\text { Intervalo } \\
\text { entre cortes } \\
\text { (dias) }\end{array}$} & \multirow{3}{*}{$\begin{array}{l}\text { Estação } \\
\text { do ano }\end{array}$} & \multicolumn{7}{|c|}{ Matéria seca de forragem $\left(\mathrm{t} \mathrm{ha}^{-1}\right)$} & \multirow{2}{*}{\multicolumn{4}{|c|}{$\begin{array}{c}\text { Matéria seca de raiz } \text { (g planta }^{-1} \text { ) } \\
\text { Condições de umidade }^{(2)}\end{array}$}} \\
\hline & & \multicolumn{3}{|c|}{ Por estação ${ }^{(1)}$} & \multicolumn{4}{|c|}{ Por corte ${ }^{(2)}$} & & & & \\
\hline & & $\mathrm{H}_{1}$ & $\mathrm{H}_{2}$ & $\mathrm{H}_{3}$ & Corte & $\mathrm{H}_{1}$ & $\mathrm{H}_{2}$ & $\mathrm{H}_{3}$ & Corte $^{(3)}$ & $\mathrm{H}_{1}$ & $\mathrm{H}_{2}$ & $\mathrm{H}_{3}$ \\
\hline 100 & Inverno & $0,0(0)$ & $1,5(9)$ & $1,8(12)$ & $\mathrm{C}_{1}$ & 0,0 & 1,5 & 1,8 & $\mathrm{C}_{1}$ & $2,89 \mathrm{a}$ & $2,91 \mathrm{a}$ & $2,16 b$ \\
\hline 34 & & & & & $\mathrm{C}_{2}$ & 0,7 & 1,3 & 1,4 & $\mathrm{C}_{2}$ & - & - & - \\
\hline 21 & & & & & $\mathrm{C}_{3}$ & 0,0 & 1,1 & 1,3 & $\mathrm{C}_{3}$ & - & - & - \\
\hline 27 & Primavera & $1,8(16)$ & $4,9(28)$ & $4,9(32)$ & $\mathrm{C}_{4}$ & 1,1 & 2,5 & 2,2 & $\mathrm{C}_{4}$ & - & - & - \\
\hline 28 & & & & & $\mathrm{C}_{5}$ & 3,4 & 3,6 & 2,0 & $\mathrm{C}_{5}$ & - & - & - \\
\hline 24 & & & & & $\mathrm{C}_{6}$ & 2,1 & 2,0 & 1,5 & $\mathrm{C}_{6}$ & - & - & - \\
\hline 27 & Verão & $7,0(61)$ & $7,3(42)$ & $5,1(34)$ & $\mathrm{C}_{7}$ & 1,5 & 1,7 & 1,6 & $\mathrm{C}_{7}$ & - & - & - \\
\hline 27 & & & & & $\mathrm{C}_{8}$ & 1,3 & 1,1 & 1,4 & $\mathrm{C}_{8}$ & - & - & - \\
\hline 36 & & & & & $\mathrm{C}_{9}$ & 1,3 & 1,4 & 0,9 & $\mathrm{C}_{9}$ & - & - & - \\
\hline 41 & Outono & $2,6(23)$ & $3,8(21)$ & $3,3(22)$ & $\mathrm{C}_{10}$ & 0,0 & 1,3 & 1,0 & $\mathrm{C}_{10}$ & $44,47 \mathrm{a}$ & $41,56 \mathrm{a}$ & $30,53 b$ \\
\hline Total & & & & & & $11,4 b$ & $17,5 \mathrm{a}$ & $15,1 \mathrm{a}$ & & & & \\
\hline
\end{tabular}

${ }^{(1)}$ Números entre parênteses representam porcentagem de matéria seca de forragem, durante as estações do ano. (2)Médias de peso da matéria seca de forragem e de raízes, seguidas da mesma letra não diferem entre si, pelo teste de Tukey a $1 \%$ de probabilidade. ${ }^{(3)} \mathrm{A}$ matéria seca da raiz só foi medida no primeiro $\left(\mathrm{C}_{1}\right)$ e no último corte $\left(\mathrm{C}_{10}\right)$ 
Na época das chuvas (primavera-verão), o rendimento dos cortes 5, 6, e 9 nos tratamentos $\mathrm{H}_{1}$ e $\mathrm{H}_{2}$ foi superior ao do tratamento $\mathrm{H}_{3}$ (Tabela 2). Ou seja, com a presença da água e com maior sistema radicular, a produtividade de forragem de alfafa foi superior Smeal et al. (1992), nos EUA, também observaram que o rendimento da alfafa aumentou em média $0,5 \mathrm{tha}^{-1}$ para cada $27,5 \mathrm{~mm}$ de água aplicada, sendo que o crescimento de raiz e a evapotranspiração potencial foram os principais condicionantes desse incremento de produção.

\section{Conclusões}

1. A irrigação da cultura da alfafa, no Latossolo Vermelho-Amarelo de textura média, pode ser realizada a partir da emissão do primeiro afilho secundário, quando as diferenças entre os valores acumulados do tanque classe $\mathrm{A}$ e da precipitação pluviométrica estiverem em torno de $30 \mathrm{~mm}$.

2. Em virtude da facilidade de obtenção dos dados, o balanço entre a evaporação do tanque $\mathrm{A} e$ as precipitações pluviais é um método prático para manejar a irrigação complementar de alfafa.

\section{Referências}

CALDERANO FILHO, B.; SANTOS, H. G.; FONSECA, O. O. M.; SANTOS, R. D.; PRIMAVESI, O.; PRIMAVESI, A. C. Os solos da fazenda Canchim. Rio de Janeiro: Embrapa-CNPS, 1998. 95 p. (Boletim de Pesquisa, 7)

FONTES, P. C. R.; MARTINS, C. E.; COSER, A. C.; VILELA, D. Produção e níveis de nutrientes em alfafa (Medicago sativa L.) no primeiro ano de cultivo, na Zona da Mata de MG. Revista da Sociedade Brasileira de Zootecnia, Viçosa, v. 22, n. 2, p. 205-211, 1993.

FRIZZONE, J. A.; TEODORO, R. E. F.; PEREIRA,A. S.; BOTREL, T. A. Lâminas de água e doses de nitrogênio na produção de aveia (Avena sativa L.) para forragem. Scientia Agricola, Piracicaba, v. 52, n. 3, p. 578-586, 1995
GILL, P. S.; MALIK, B. S. Response of oat varieties to soil moisture regimes and nitrogen levels. Forage Research, New Delhi, v. 9, n. 2, p. 151-154, 1983.

GILLEY, J. R.; HEERMANN, D. F.; STETSON, L. E. Irrigation management energy. In: ASAE NATIONAL IRRIGATION SYMPOSIUM, 2., 1981, Lincoln. Proceedings... Lincoln: ASAE, 1981. p. 127-140.

HUKKERI, S. B.; SHUKLA, N. P.; RAJPUT, R. K. Effects of levels of soil moisture and nitrogen on the fodder yield of oat on two types of soil. Indian Journal of Agricultural Sciences, New Delhi, v. 47, n. 4, p. 204209, 1977

KLAR, A. E. Irrigação: freqüência e quantidade de aplicação. São Paulo: Nobel, 1991. 156 p.

LAL, M. Studies on irrigation scheduling in nited stands of forage oat and legumes. Indian Agronomy Journal, Madison, v. 32, n. 1, p. 21-23, 1987

PIMENTEL-GOMES, F. Curso de estatística experimental. 8. ed. Piracicaba: Esalq, 1978. 430 p.

PRIMAVESI, O.; PRIMAVESI, A. C. P. A.; PEDROSO, A. F.; CAMARGO, A. C.; RASSINI, J. B.; ROCHA FILHO, J.; OLIVEIRA, G. P.; CORREA, L. A.; ARMELIN, M. I. A.; VIEIRA, S. R.; DECHEN, S. C. F. Microbacia hidrográfica do ribeirão Canchim: um modelo real de laboratório ambiental. São Carlos: EmbrapaCPPSE, 1999. 133 p. (Boletim de Pesquisa, 5).

RASSINI, J. B. Alfafa (Medicago sativa L.): estabelecimento e cultivo no Estado de São Paulo. São Carlos: Embrapa-CPPSE, 1998. 22 p. (Circular Técnica, 15)

RASSINI, J. B.; LEME, E. J. A. Water management for establishment of alfalfa. In: INTERNATIONAL GRASSLAND CONGRESS, 19., 2001, São Pedro. Proceedings... São Pedro: Sociedade Brasileira de Zootecnia, 2001. p. 260-261

SANTOS, J. A. Alfafa é mais proteína e energia a custo menor. Balde Branco, São Paulo, v. 43, p. 30-34, dez. 1993

SMEAL, D.; KALLSEM, C. E.; SAMMIS, T. W. Alfalfa yields as related to transpiration, growth stage and environment. Irrigation Science, Berlin, v. 12, n. 2, p. 79 $86,1992$. 